\title{
First measurements with the K-monochromator at the European XFEL
}

\section{Wolfgang Freund, Lars Fröhlich, Suren Karabekyan, Andreas Koch, Jia Liu, Dirk Nölle, Josef Wilgen and Jan Grünert}

J. Synchrotron Rad. (2019). 26, 1037-1044

\section{IUCr Journals CRYSTALLOGRAPHY JOURNALS ONLINE}

Copyright (C) International Union of Crystallography

Author(s) of this article may load this reprint on their own web site or institutional repository provided that this cover page is retained. Republication of this article or its storage in electronic databases other than as specified above is not permitted without prior permission in writing from the IUCr.

For further information see http://journals.iucr.org/services/authorrights.html 
JOURNAL OF

SYNCHROTRON

RADIATION
Received 21 December 2018

Accepted 16 April 2019

Edited by D. Cocco, SLAC National Accelerator Laboratory, USA

${ }^{1}$ This article will form part of a virtual special issue containing papers presented at the PhotonDiag2018 workshop.

Keywords: XFEL; K-mono; undulator adjustment.

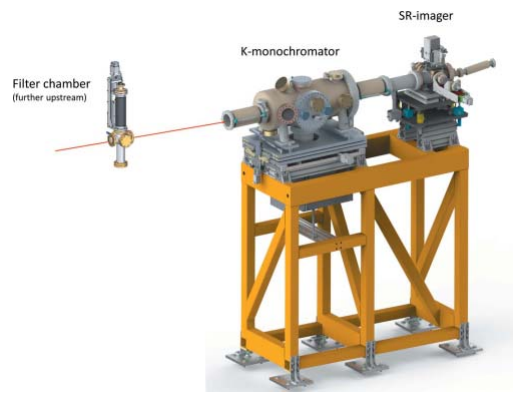

(C) 2019 International Union of Crystallography

\section{First measurements with the K-monochromator at the European XFEL ${ }^{1}$}

\author{
Wolfgang Freund, ${ }^{\mathrm{a} *}$ Lars Fröhlich, ${ }^{\mathrm{b}}$ Suren Karabekyan, ${ }^{\mathrm{a}}$ Andreas Koch, ${ }^{\mathrm{a}}$ Jia Liu, \\ Dirk Nölle, ${ }^{b}$ Josef Wilgen ${ }^{b}$ and Jan Grünert ${ }^{a}$
}

${ }^{\mathbf{a}}$ European X-ray Free Electron Laser Facility GmbH, 22869 Schenefeld, Germany, and ${ }^{\mathbf{b}}$ Deutsches ElektronenSynchrotron DESY, 22607 Hamburg, Germany. *Correspondence e-mail: wolfgang.freund@xfel.eu

Hard X-ray free-electron lasers (XFELs) generate intense coherent X-ray beams by passing electrons through undulators, i.e. very long periodic magnet structures, which extend over hundreds of meters. The SASE1 and SASE2 undulator systems of the European XFEL consist of 35 segments with variablegap planar undulators which are initially tuned to precise on-axis magnetic field strengths in a magnetic measurement laboratory to keep an important quality parameter - the $K$-value variation from segment to segment - below a certain limit $\left(3 \times 10^{-4}\right.$ for $12 \mathrm{keV}$ photon energy). After tunnel installation only photon-based methods can determine the $K$-values of undulator segments with a similar accuracy. The synchrotron radiation from a single or a few segments can be spectrally filtered by a dedicated crystal monochromator (K-monochromator) and recorded with a photodiode or with an imager that provides 2D information, tuned for high sensitivity to detect low photon densities from distant single undulator segments. This instrumentation is applied for electron orbit analysis and optimization, and adjustment of individual undulators in terms of their central magnetic axis with respect to the electron beam. Single undulator segments were analysed by scanning the monochromator crystal angle and detecting the steepest slope of a photodiode signal. Alternatively, in the imaging method, an imager recorded the radiation cone of electrons passing through the undulator segment. From the spatial distribution of the radiation, the $K$-parameter was determined with a sufficiently high relative accuracy.

\section{Introduction}

The European X-ray Free Electron Laser (EuXFEL) has two hard X-ray self-amplified spontaneous emission (SASE) undulator systems and one soft X-ray SASE undulator system for producing high-brightness laser radiation. A beam transportation system delivers the beam to, in the final stage of completion, six scientific instruments (Tschentscher et al., 2017).

The hard X-ray undulators SASE1 and SASE2 consist of 35 undulator segments, each $5 \mathrm{~m}$ long with a $40 \mathrm{~mm}$ magnet period, separated by $1.1 \mathrm{~m}$-long intersections for e-beam steering, focusing and phase adaptation. For the case of the soft X-ray undulator (SASE3) the setup is similar but has 21 segments and a longer magnet period of $68 \mathrm{~mm}$.

One prerequisite for achieving lasing is the tuning of the $K$-value of all undulator segments to a very high precision. The undulator segments are characterized by the $K$-parameter, $K=\left(e B \lambda_{\mathrm{u}}\right) /\left(2 \pi m_{\mathrm{e}} c\right)$, where $B$ is the amplitude of the on-axis magnetic field and $\lambda_{\mathrm{u}}$ is the undulator period.

From FEL tolerance calculations it can be shown that the relative error in the produced wavelength must be smaller than the Pierce parameter $\rho . \rho$ is a fundamental scaling parameter and gives a measure of the exponential gain and 
saturated efficiency of a high-gain FEL, with typical values in the X-ray regime of $10^{-4} \leq \rho \leq 10^{-3}$ (Tischer et al., 2002; McNeil \& Thompson, 2010). The error in $K$ for the given error in $\lambda$ at $1 \AA(\sim 12 \mathrm{keV}$ photon energy) is approximately $3 \times 10^{-4}$ and determines the required $K$-measurement accuracy. This magnetic tuning and calibration of the undulators was performed in the magnetic laboratory before transporting to the final position in the tunnels. Mounted in the tunnels there is no possibility to measure the $K$-values precisely enough by means of magnetic measurements. Photon-based commissioning (Grünert, 2012; Grünert et al., 2019) of the European XFEL undulators requires a precise adjustment of the $K$-parameters of all undulator segments and phasing between these segments. The undulator commissioning spectrometer, also known as the K-monochromator or K-mono, makes it possible to measure the spontaneous radiation of the undulator segments for $K$-tuning and other diagnostic measurements (e.g. pointing, phase matching) (Tischer et al., 2002; Tanaka, 2009; Welch et al., 2009).

One important goal is the characterization of the undulator segments with a well defined electron orbit after electronbeam-based alignment and once lasing has been established. In this case a database of energy spectrum measurements for each undulator segment will make it possible to identify changes due to radiation damage or changes in the mechanical alignment.

\section{Description of the K-mono system}

The K-monochromator system, which was developed at the European XFEL (Ozkan et al., 2012; Freund, 2013; Rehanek, 2014), consists of three devices: the filter chamber for inserting various metal foils into the beam, the K-monochromator with two independent goniometer stages, and the spontaneous radiation imager (SR-imager) as the detection unit (Fig. 1) (Koch et al., 2015).

The filter chamber contains a linear manipulator with five positions for filter holders which have an aperture of $30 \mathrm{~mm}$. It is equipped with $3 \mu \mathrm{m} \mathrm{Al}$ for blocking visible light, and different foils ( $\mathrm{Ni}, \mathrm{Cu}, \mathrm{Mo}$ ) for $K$-edge energy calibrations.

The power slit (SRA) between filter chamber and K-mono is the only X-ray optical element upstream of the K-mono. Usually this slit is widely open for K-mono measurements and only blocks the upper part of the beam which could otherwise pass over the first crystal and the beamblocker of the K-mono.

The K-mono itself consists of two independent goniometer stages with absolute encoders, equipped with $\mathrm{Si}(111)$ channelcut crystals (Fig. 2). The Bragg angle covers the range $7-55^{\circ}$ which corresponds to an energy range of $2.5-16 \mathrm{keV}$ (Si 111) or $7.5-48 \mathrm{keV}$ in the case of the $\mathrm{Si}(333)$ reflection. The energy resolution is $\Delta E / E=2 \times 10^{-4}$ for $\operatorname{Si} 111\left(1 \times 10^{-5}\right.$ for Si333). The second crystal can be rotated out of the beam for switching from four-bounce mode (Bartels geometry) to twobounce mode. For complete retraction the whole chamber can be moved horizontally, so that the beam passes crystals and beamblocker on the side. The photon energy range for the SASE1 and SASE2 undulators is $3-25 \mathrm{keV}$ (core range $5-18 \mathrm{keV}$ ) which can be covered by the crystal reflections. The photon energy range for the soft X-ray undulator SASE3 is $0.28-3 \mathrm{keV}$ (core range $0.4-2 \mathrm{keV}$ ), which cannot be transmitted over the silicon crystal, and the fundamental of the spontaneous radiation is too divergent to pass through the aperture given by the beam pipe. Therefore, in the case of SASE3, the third to the ninth harmonic (or even higher harmonics) of the spontaneous radiation must be used for the measurements.

The K-mono system was designed for spontaneous radiation. However, in the case of the hard X-ray undulators SASE1 and SASE2, FEL measurements are also possible and were already tested. Due to the very low content of harmonic radiation in the FEL beam, the K-mono cannot be used for soft X-ray FEL measurements below $2.5 \mathrm{keV}$ at the SASE3 undulator, since the beam is not transmitted through the $\mathrm{K}$ mono and the filter foil.
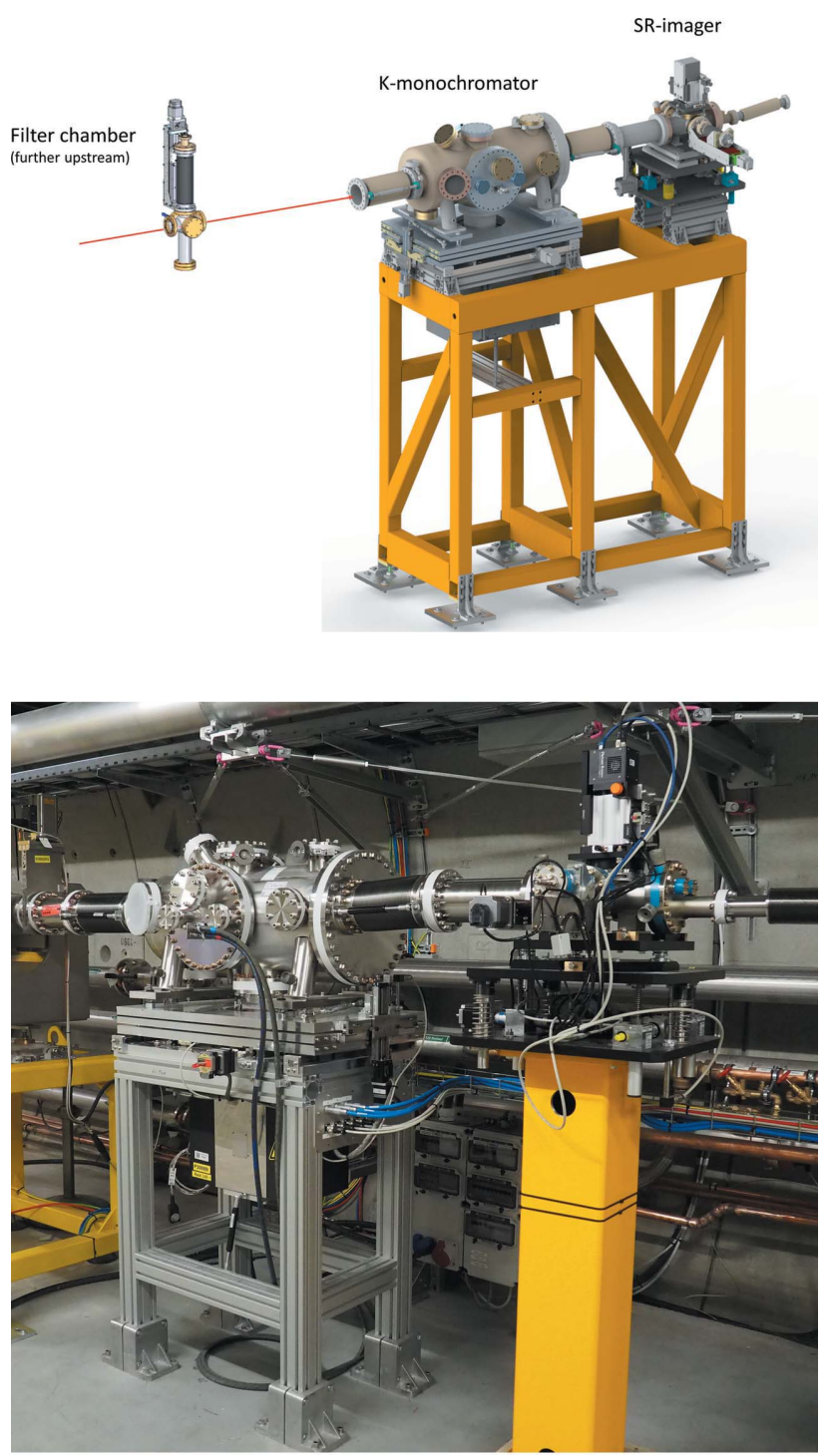

Figure 1

Top: schematic of the setup in the SASE2 tunnel: filter chamber, K-mono, SR-imager. Bottom: photograph of the K-mono and SR-imager in the SASE3 beamline. 


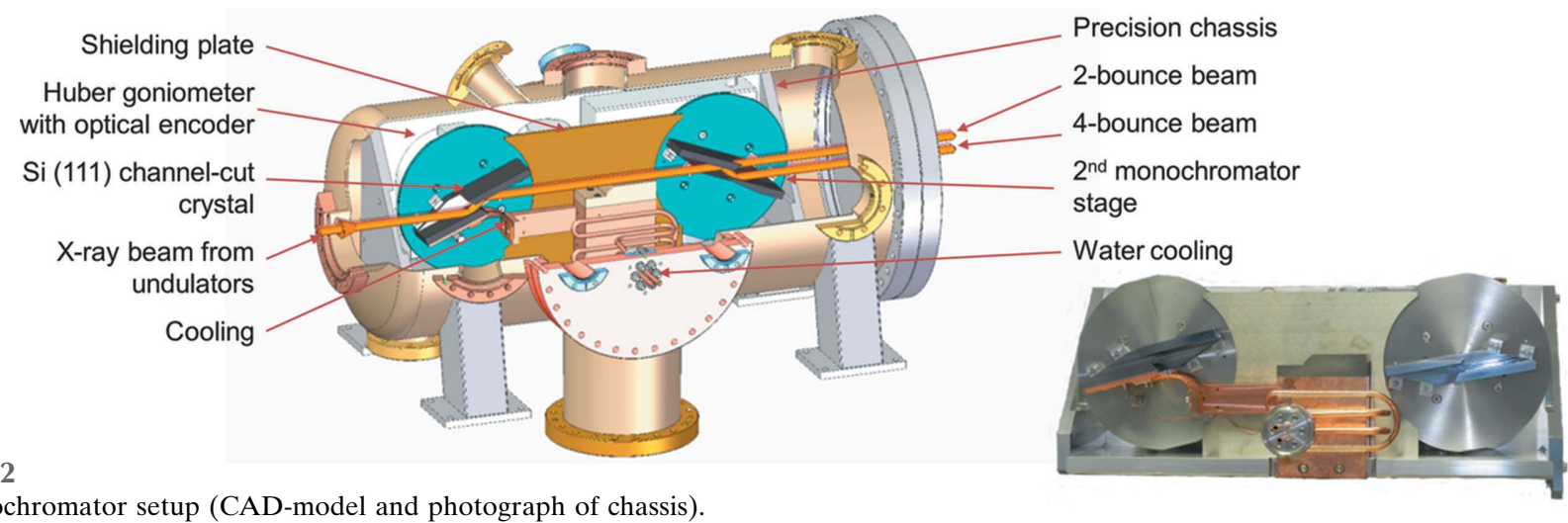

K-monochromator setup (CAD-model and photograph of chassis).

The geometry of the system is optimized for observing spontaneous radiation, which has higher divergence compared with the FEL beam. Thus the acceptance angle for the incident beam is greater than $20 \mu \mathrm{rad}$. The beam spot at the entrance of the K-mono can be 10-12 $\mathrm{mm}$ high and up to $26 \mathrm{~mm}$ wide to allow for accepting two horizontally separated beam spots.

The SR-imager (Fig. 3) is located directly downstream of the K-mono. In order to allow even single or few-pulse measurements it was designed for a maximum light yield (see specification table). A 16-bit camera with $1920 \times 1080$ pixels is equipped with a tandem lens system with a relatively short working distance to the Gd2O2S:Pr (GOS) or Y3A15O12:Ce (YAG) scintillator. The scintillators can be exchanged with a linear manipulator, which can also move a silicon PIN diode into the beam. A lifting platform can move the imager vertically into three distinct positions: at the lowest position the imager is in the direct beam or ready for four-bounce mode. In an intermediate position at approximately $20 \mathrm{~mm}$ offset the two-bounce beam is accepted. The highest position is the safe out-of-beam position.

\section{Measurements}

The goal of the measurement described in this paper was the adjustment of the SASE1 undulator using the K-mono mainly with the SR-imager. Other methods and preparatory test measurements using the photodiode or the dipole kick procedure are described by Freund et al. (2019) and Ozkan et al. (2012).

For practical reasons (e.g. available beam time) we decided to concentrate mainly on relative measurements and did not use the filter foils with their $K$-edges for an absolute energy calibration. However, there are sharp monochromator glitches in the spectra which can be used for determination of the absolute encoder offset. These glitches are caused by higherorder crystal reflections under different angles than the main reflection which therefore do not pass the second crystal surface. We have calculated the glitch reflections with the software package XOP (Sanchez del Rio \& Dejus, 2004) and identified the glitches fingerprint in the raw data (Fig. 4). As there is some uncertainty about the crystal miscut and azimuthal angle, the final calibration will be later crosschecked using the filters.

We also performed a calibration by using the undulator harmonics, which is especially useful in the soft X-ray domain, where many more harmonics can be observed (shown later in Figs. 10 and 11). Measurements for the hard X-ray undulator SASE2 from the fundamental at $6.6 \mathrm{keV}$ to the fifth harmonic show also good coincidence with the glitch determination.

Prerequisite for good measurements of undulator parameters is a well defined electron orbit (the same that is established for lasing). In order to achieve the required high

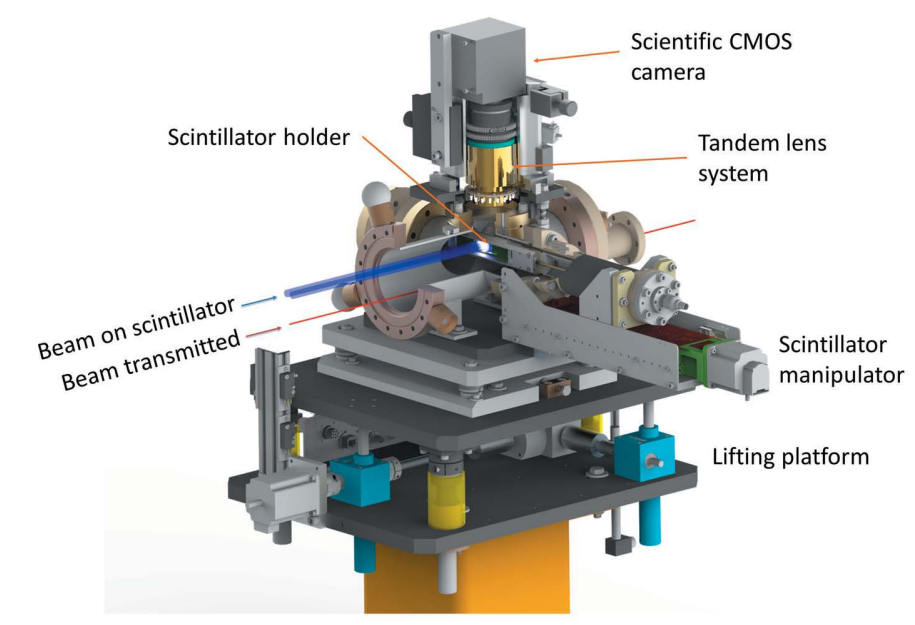

SR-imager specifications

- Sensitive sCMOS camera: FullHD 2 Mpixel Readout noise $0.5 \mathrm{e}-/ \mathrm{s}$ dark current: $0.9 \mathrm{e}-$

- Tandem lens magnification 0.47

- eff. $\mathrm{F}_{\infty}$-number 0.95

- Field of view: $27 \times 15 \mathrm{~mm}^{2}$

- Pixel size on screen: $14 \mu \mathrm{m}$

- PIN diode: $10 \times 10 \mathrm{~mm}^{2}$

- Noise equivalent signal: 1 photon@ 3 keV (with GOS scintillator)

Figure 3

CAD-model of the SR-imager. 


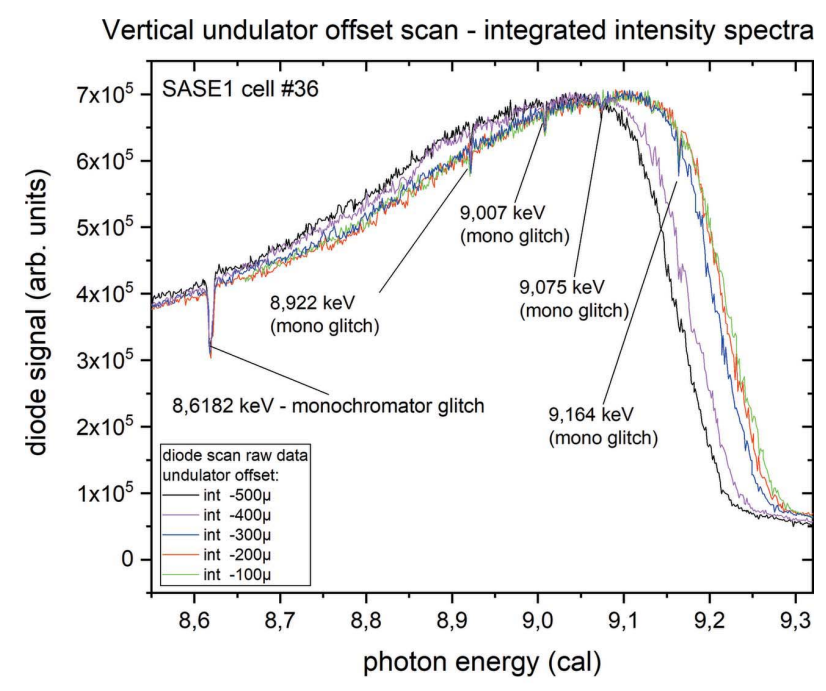

XOP MARE-Glitches, Main diffraction: $[1,1,1]$ Si 90 deg

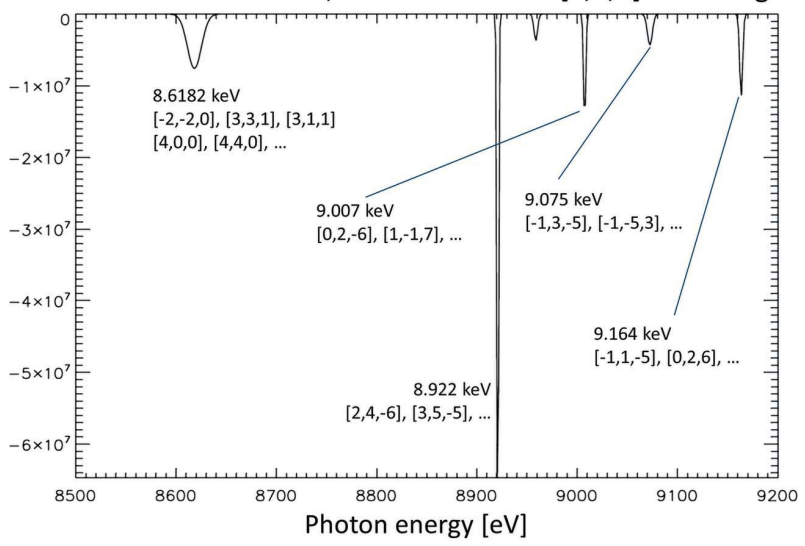

Figure 4

Top: integrated intensity scans for various vertical offsets of the undulator centre - raw spectra exhibiting monochromator glitches. Bottom: XOP calculation with the MARE module.

peak currents for lasing, the electron bunch is compressed using magnetic chicanes. This requires an energy chirp which smears out the spectra and images. Usually the effect is not as strong as shown in Fig. 5, but it is always visible and varies with electron beam condition and undulator position. When the electron beam is well established (e.g. just before or after a user run) it is possible to acquire sensible results with a fully compressed beam, while during the start-up phase after a shutdown the ring fitting algorithm can fail. In order to avoid these effects, the measurements are usually performed with reduced compression of the electron beam.

\section{Method}

There are several methods for $K$-tuning, phase matching and trajectory alignment for which the K-mono can be used (Tischer et al., 2002; Tanaka, 2009; Welch et al., 2009). Here we have used the K-mono with SR-imager for observing the spatial profile of the spontaneous radiation of single undulator segments, which during our tests appeared to be the fastest method.

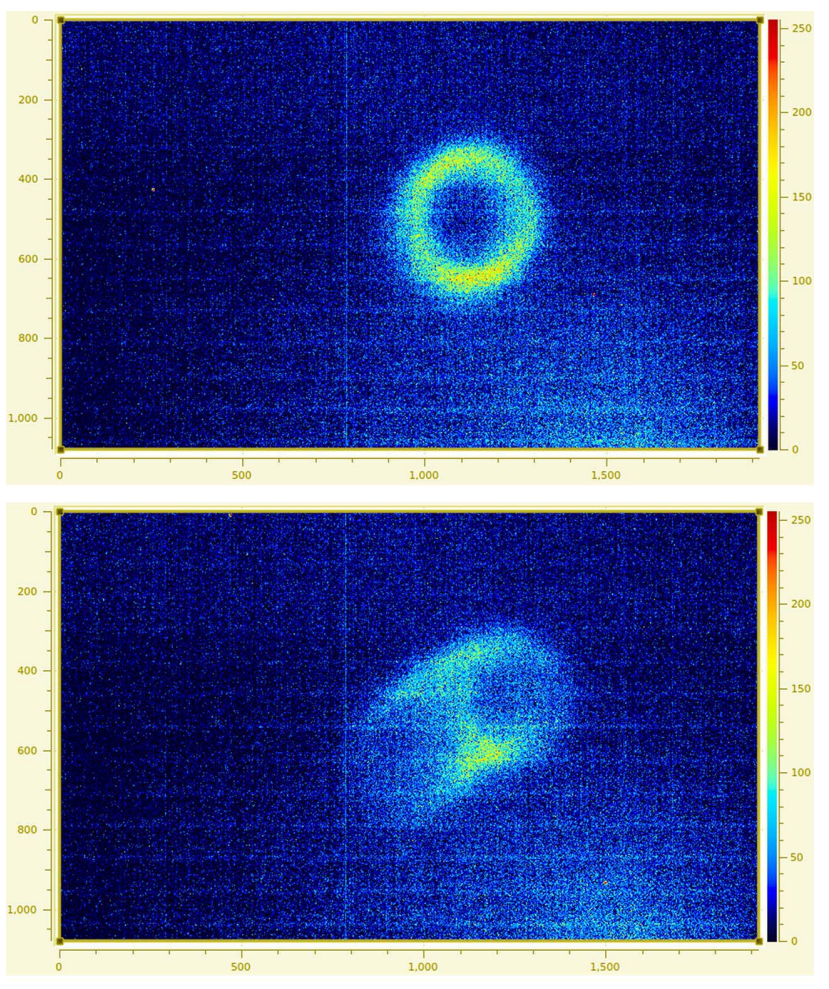

Figure 5

Ring structure with uncompressed electron beam (top) and compressed beam (bottom).

When tuning the K-mono to photon energies slightly below the resonant energy of the undulator segments, donut-like rings appear, as the divergence angle of the spontaneous radiation depends on the photon energy and the off-axis spectrum becomes 'red-shifted'. For even harmonics the onaxis flux is zero, but flux can be observed at an angle of only a few microradians away from the axis.

The diameters of the rings depend on the gap settings, e-beam energy, detuning factor and distance to the undulator segment. With these parameters known with high accuracy, the $K$ parameter can be determined directly from the ring diameter, which is a measure for the observation angle $\theta$ in the undulator equation,

$$
\lambda_{n}=\frac{\lambda_{\mathrm{u}}}{2 n \gamma^{2}}\left(1+\frac{K^{2}}{2}+\theta^{2} \gamma^{2}\right) .
$$

For relative $K$ tuning of the segments it is sufficient to tune to the same spatial profile by taking into account the geometrical factor of different distance to the screen, since all other parameters are equal for the segments. When looking more precisely, there is a slight distortion in the vertical direction, and therefore a slightly larger vertical ring diameter due to the two-bounce mode of the monochromator with the dispersion in the vertical direction. Due to the large distance to the source point, the acceptance angle of the monochromator crystal is small $(10-20 \mu \mathrm{rad})$ and this effect can be neglected. For very precise measurements the beam should always be vertically centred on the screen, in order to avoid small energy 


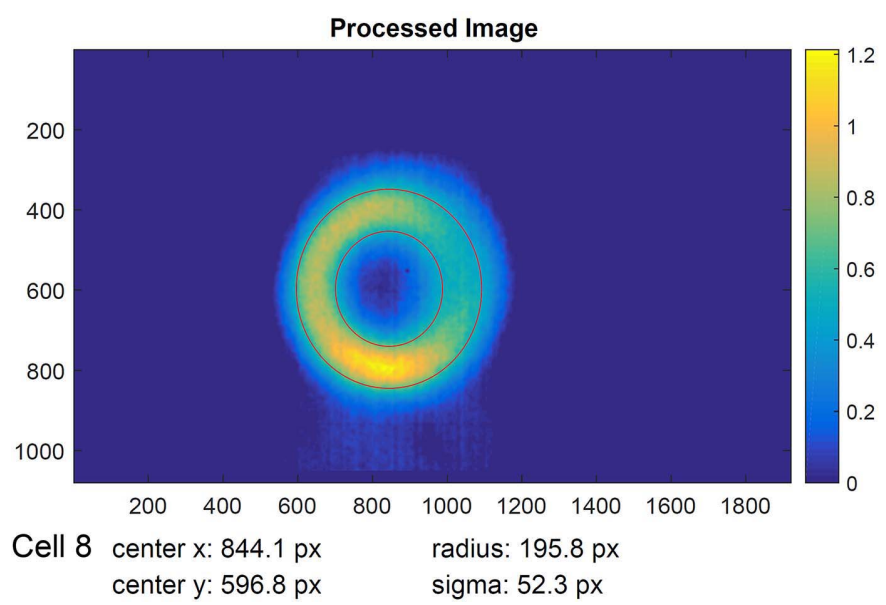

Figure 6

Example of online ring fitting, here for undulator segment 8 of SASE1.

shifts. From the rings, the spatially integrated intensity and the beam pointing can also be determined.

\section{SASE1 undulator orbit correction}

K-mono measurements provide information about the electron beam which is supplementary to the electron beam diagnostics data. The distance from the undulator segment to the imager is large (between 180 and $400 \mathrm{~m}$ ), therefore the beam position on the SR-imager screen is a direct measure for the angular pointing of the e-beam inside the undulator segment. The effect of the e-beam offset is almost negligible and can be better observed with the e-beam diagnostics. Since the effects of adjustment of air coils, quadrupole movers or undulator gap can be directly seen on the SR-imager, this is a very intuitive tuning method to check and fine-adjust the electron beam in the undulator section.

\section{SASE1 K-adjustment}

For the SASE1 undulator we have performed three complete scans where segment by segment was closed to the nominal $K$ value for $9.3 \mathrm{keV}$ photon energy while all other segments were opened. The fitting algorithm was applied to the images to determine the ring diameters and subsequently the $K$-values (Fig. 6). Two independent measurements (repeated after $2 \mathrm{~h}$ ) were performed in July in order to estimate the measurement error, including drifts of e-beam energy and position as well as electronic noise. This scan was repeated in October after the vertical alignment of cell 8 (green curve in Fig. 7).

The slight slope in the measurements from July is caused by a residual linear taper setting along the undulator system. The correlation of the curves is quite good, considering that three months passed between the measurements and beam parameters change. Most notably the deviation at cell 8 has vanished after the vertical alignment (described in the next section). The measurement for cell 3 , which is the first cell of the undulator, is not reliable, since there was no clear ring image. These measurements show that the r.m.s. value of $\Delta K / K$ for the entire SASE1 system, without middle plane adjustment and excluding applied linear taper, is $\sim 4.3 \times 10^{-4}$.

An important improvement for the latest measurements was an automated routine for driving the undulator gaps, taking the images and fitting the ring diameters. With this the measurement time for scanning all 35 segments of the undulator could be reduced to less than $45 \mathrm{~min}$.

\section{SASE1 vertical undulator position adjustment}

From tuning of lasing in the SASE1 undulator system it was suspected that the vertical position (i.e. the magnetic middle plane) of undulator segment 8 was not centred with respect to the electron beam. This suspicion was verified with a procedure for finding the vertical magnetic centre of the undulator gap.

The vertical deviation from the presumed magnetic axis of the undulator ( $y$-offset) was varied in steps of $100 \mu \mathrm{m}$ while keeping the gap constant. In the actual vertical centre of the undulator gap the magnetic field (and with it the $K$ value) is minimal, thus the resonance photon energy is maximized [see Table 1 (Pflüger, 2018)]. The K-mono is tuned below the undulator resonance, therefore the difference between resonance energy and K-mono energy is highest and thus the ring diameter is largest.

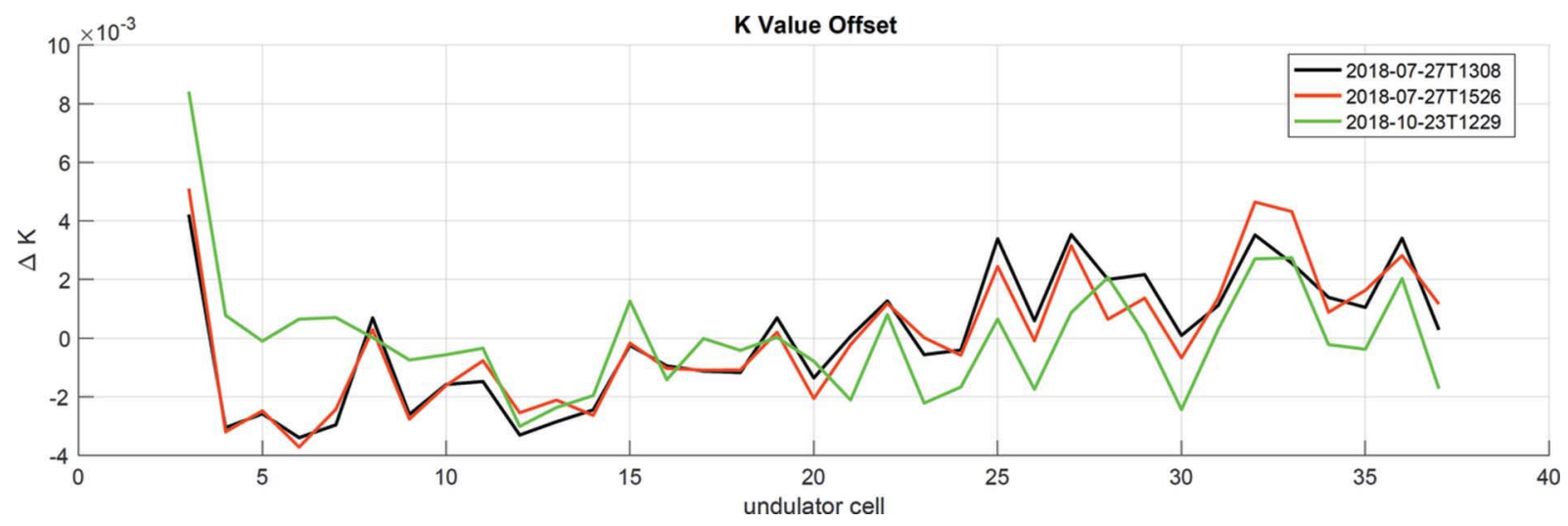

Figure 7

SASE1 $K$-scans. 
Table 1

$\Delta K / K$ variation along $y$ (Pflüger, 2018).

\begin{tabular}{ll}
\hline $\begin{array}{l}\text { Offset from } \\
\text { centre, } y(\mathrm{~mm})\end{array}$ & $\begin{array}{l}\text { Resulting deviation from } \\
\text { nominal } K, \Delta K / K^{\dagger}\end{array}$ \\
\hline 0 & 0 \\
0.1 & 0.000123 \\
0.2 & 0.000493 \\
0.3 & 0.001110 \\
0.4 & 0.001973 \\
0.5 & 0.003084 \\
\hline
\end{tabular}

$\dagger \Delta K / K=(1 / 2)\left(2 \pi y / \lambda_{\mathrm{u}}\right)^{2}$, where $\lambda_{\mathrm{u}}=40 \mathrm{~mm}$.

SASE1 cell \#8 - vertical undulator offset scan

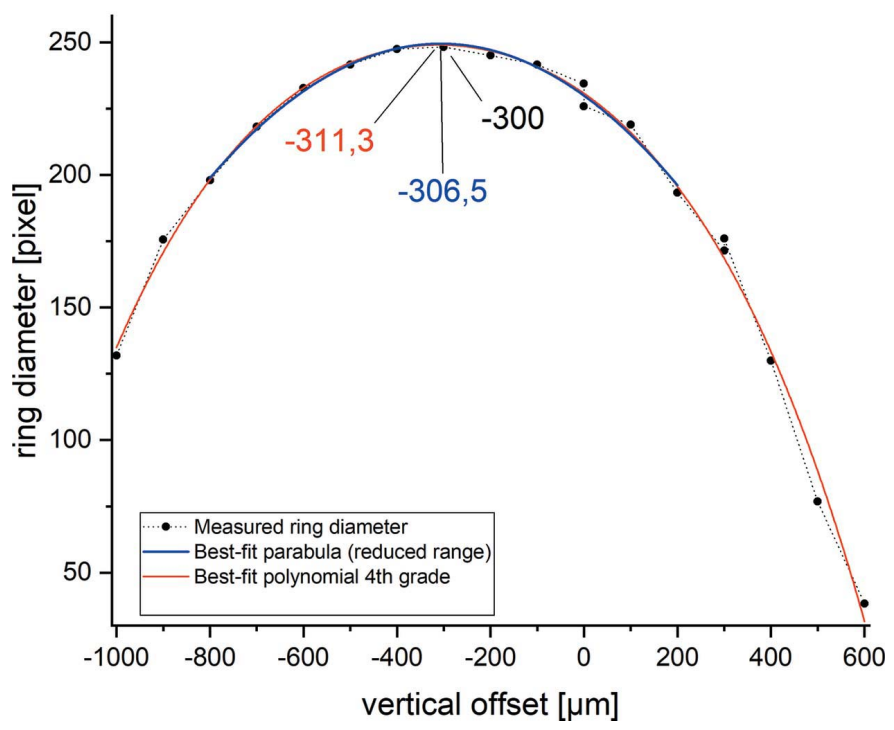

Figure 8

Ring radius versus vertical undulator offset.

From Table 1 we can derive that a vertical offset of $200 \mu \mathrm{m}$ from the centre corresponds to a deviation of $K$ in the order of the Pierce parameter. With the measured data and the best-fit curves (Fig. 8) we could determine the best vertical undulator position to $-306 \mu \mathrm{m}$ offset with an estimated positioning error of less than $100 \mu \mathrm{m}$ from the centre, which is better than the requirement. It is difficult to determine an error bar for each measurement point. The two points at the zero position were measured before and during the scan. A possible reason for the deviation could be the electron beam condition, since the beam energy as well as orbit jitter (e.g. from feedback control) were not taken into account in this measurements. The undulator middle plane control accuracy could also be a reason of this. For each measurement point we have recorded more than 100 images.

When using a 2D Gaussian fitting algorithm on different samples of this data set we obtain only small errors (for sufficiently large rings greater than 100 pixels) which could not explain the deviation.

For an earlier measurement campaign we performed energy scans with the K-mono and recorded a set of integrated intensity scans with the diode for various $y$-offsets. Unfortunately, we could not vary the offset for the needed range due

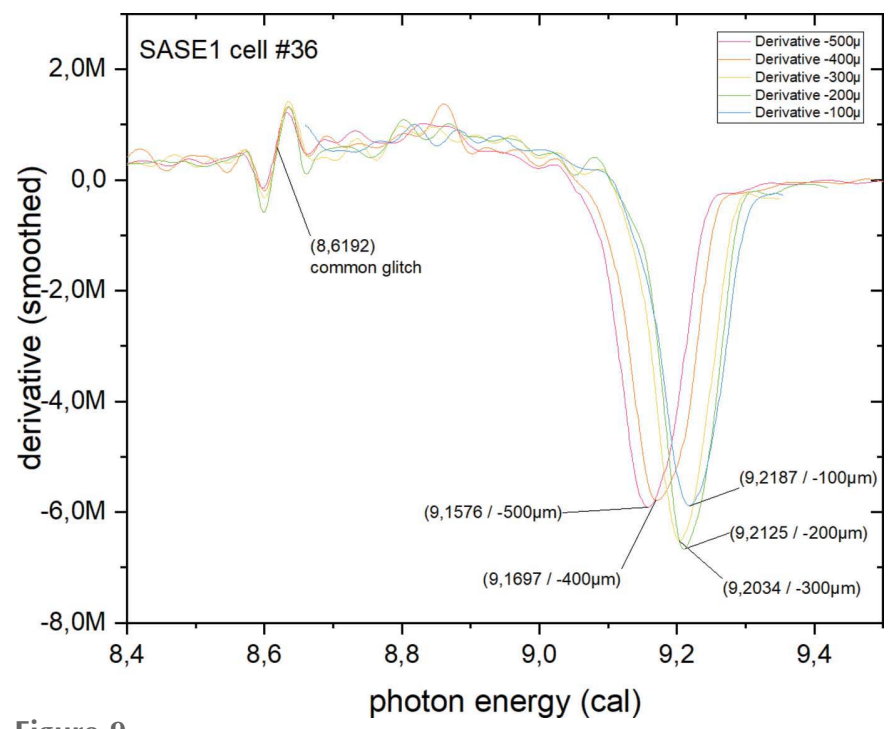

Figure 9

Derivatives of integrated intensity scans for various vertical offsets of the undulator centre from Fig. 4.

to technical limitations during the beam time. The set of curves (Fig. 4) that we were able to acquire were used to determine the fundamental energy of one undulator cell at different vertical offset positions. At the high-energy edge of the spectrum, which is at the minimum of the derivative (Fig. 9), it is possible to distinguish different resonance energies for a difference of $100 \mu \mathrm{m}$ vertical offset close to the magnetic centre. For a more accurate error analysis we could not take enough data, but we now think that both methods show a similar (sufficient) accuracy for the determination of the $K$-parameter and therefore the magnetic centre.

Compared with the imaging method a scan of the goniometer takes a few minutes, which is notably more time than the fraction of a second to record the image, where no mechanical motion is needed.

\section{K-mono scan of SASE3 undulator segment (cell 23)}

This measurement was performed in order to test and commission the SASE3 K-monochromator system and for energy calibration. The spectral scan was performed by scanning the Bragg angle of the first crystal, while the second crystal was rotated out of the beam (two-bounce mode). The goniometer was moved continuously over the measurement range, while an image and all relevant slow parameters were acquired at $10 \mathrm{~Hz}$ for each pulse train, for which each was filled with 30 pulses. With the large Bragg angle scan range from $46^{\circ}$ to $5.4^{\circ}$, corresponding to a photon energy range of $2.7 \mathrm{keV}$ to $21 \mathrm{keV}$, the $3 \mathrm{rd}$ to $23 \mathrm{rd}$ harmonics of the fundamental at approximately $900 \mathrm{eV}$ (set value) were measured. As mentioned in Section 4, the fundamental and second harmonic are not transmitted through the filter foil $(3 \mu \mathrm{m} \mathrm{Al})$ and the K-mono and were not recorded. After background subtraction we derived a video from the image scan (see Fig. 10). 

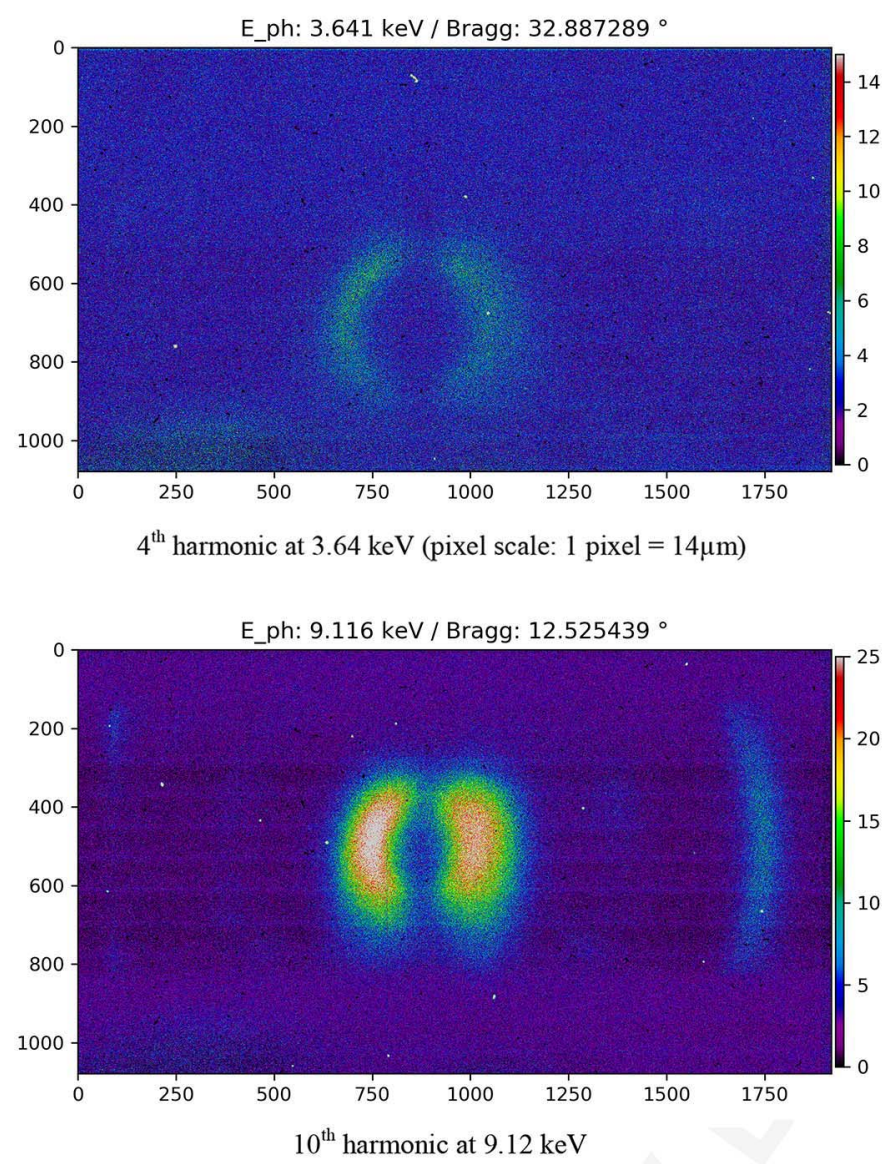

Figure 10

Selected images from the K-mono scan.

We derived an angle-integrated intensity scan by summing over the pixels of the horizontal central region of interest between pixel number 500 and 1250 . The resulting undulator spectrum is shown in Fig. 11. An absolute energy calibration was established with a least-squares fit of the high-energy edge

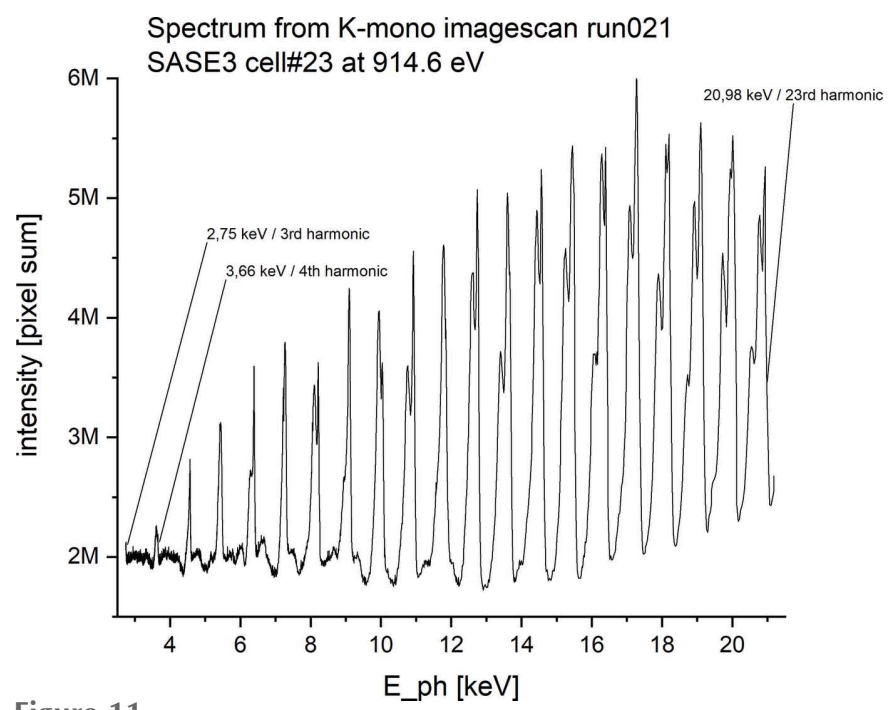

Figure 11

Angle-integrated intensity scan - undulator spectrum derived from the images.
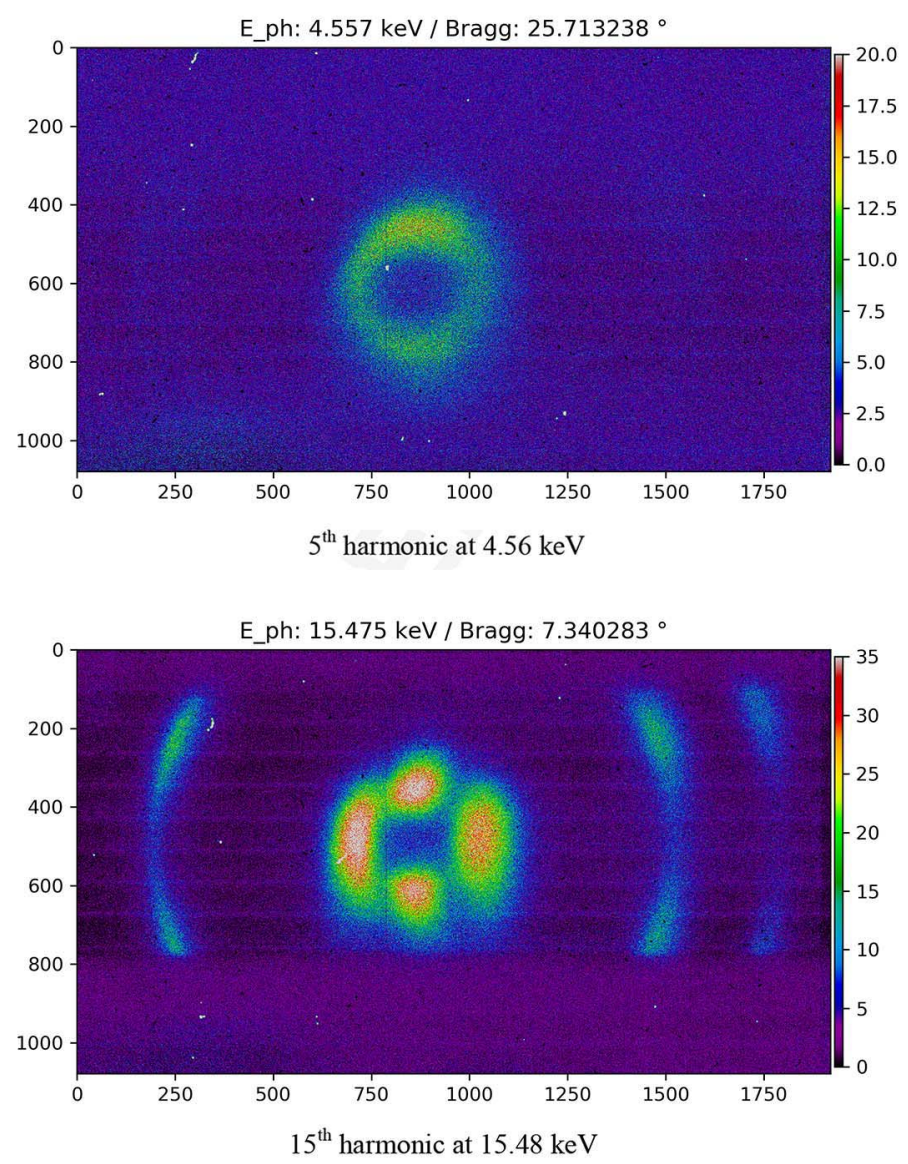

positions, and the fundamental photon energy was determined as $914.6 \mathrm{eV}$.

\section{Summary and outlook}

The K-monochromator system has proven very helpful for fine tuning of the undulator systems and for improving photon beam quality. Fast measurements for undulator adjustments are possible with the imaging methods and fitting of the spontaneous radiation spatial distribution. The K-monochromator system has proven the potential for improvement of beam orbits, determination of $K$-parameter offsets between adjacent undulator segments and for their vertical position alignment.

We have shown that the hardware of the K-monochromator system works precisely and reliably. The mechanics are stable and reproducible and the detection is sensitive enough to detect even single-pulse spontaneous radiation from individual segments (pulse-resolution might not be necessary thanks to the excellent accelerator stability within a pulse train). From diode scans as well as from the spatial distribution of the radiation we can deduce the information for adjusting the undulators with high precision. First automated procedures were used to scan all 35 segments of the SASE1 and SASE2 undulators and acquire a full dataset in a reasonably short time of less than $45 \mathrm{~min}$. There is still a large potential for 
improvements by optimization of the procedures in terms of speed, accuracy and functionality. It is planned to use the system for regular undulator inspections, e.g. for assessing radiation damages to the undulators, for which we mainly need improvements on the software side. Procedures will be further developed for automated $K$ - and hard X-ray FEL spectrum measurements.

\section{Acknowledgements}

The authors thank the experts of the European XFEL scientific support groups CAS, AE, ITDM and the DESY colleagues on the accelerator side.

\section{References}

Freund, W. (2013). Technical Note DESY-2014-03060, XFEL.EU TN2014-001-01. European XFEL, Hamburg, Germany.

Freund, W., Grünert, J., Liu, J., Karabekyan, S. \& Koch, A. (2019). AIP Conf. Proc. 2054, 030018.

Grünert, J. (2012). European XFEL Technical Report XFEL.EU TR2012-003. European XFEL, Hamburg, Germany.

Grünert, J., Planas, M., Dietrich, F., Freund, W., Koch, A., Kujala, N., Laksman, J., Liu, J. \& Maltezopoulos, T. (2019). AIP Conf. Proc. 2054, 030014.
Koch, A., Freund, W., Grünert, J., Planas, M., Roth, T., Samoylova, L. \& Lyamayev, V. (2015). Proc. SPIE, 9512, 95121R.

McNeil, B. W. J. \& Thompson, N. R. (2010). Nat. Photon. 4, 814821.

Ozkan, C., Freund, W., Rehanek, J., Buck, J., Zizak, I., Gruenert, J., Schaefers, F., Erko, A. \& Molodtsov, S. (2012). Proc. SPIE, 8504, $85040 \mathrm{X}$.

Pflüger J. (2018). Private communication and European XFEL internal report No. WP71/2017/14.

Rehanek, J. (2014). Dissertation. Freie Universität Berlin, Germany (https://refubium.fu-berlin.de/handle/fub188/9969).

Sanchez del Rio, M. \& Dejus, R. J. (2004). Proc. SPIE, 5536, 171174.

Tanaka, T. (2009). (2009). Proceedings of the 2009 Free Electron Laser Conference (FEL2009), 23-28 August 2009, Liverpool, UK, pp. 524-527. WEPC11.

Tischer, M., Ilinski, P., Hahn, U., Pflüger, J. \& Schulte-Schrepping, H. (2002). Nucl. Instrum. Methods Phys. Res. A, 483, 418-424.

Tschentscher, T., Bressler, C., Grünert, J., Madsen, A., Mancuso, A. P., Meyer, M., Scherz, A., Sinn, H. \& Zastrau, U. (2017). Appl. Sci. 2017, 7(6), 592.

Welch, J., Brachmann, A., Decker, F.-J., Ding, Y., Emma, P., Fisher, A., Frisch, J., Huang, Z., Iverson, R., Loos, H., Nuhn, H.-D., Stefan, P., Ratner, D., Turner, J., Wu, J., Xiang, D., Bionta, R. \& Sinn, H. (2009). Proceedings of the 2009 Free Electron Laser Conference (FEL2009), 23-28 August 2009, Liverpool, UK, pp. 730-733. THOA05. 